\title{
Variation of Biophysical Parameters of the Skin with Age, Gender, and Body Region
}

\author{
Alireza Firooz, ${ }^{1}$ Bardia Sadr, ${ }^{1}$ Shahab Babakoohi, ${ }^{1}$ \\ Maryam Sarraf-Yazdy, ${ }^{1}$ Ferial Fanian, ${ }^{1}$ Ali Kazerouni-Timsar, ${ }^{1}$ Mansour Nassiri-Kashani, ${ }^{1}$ \\ Mohammad Mehdi Naghizadeh, ${ }^{2}$ and Yahya Dowlati ${ }^{1}$
}

\author{
${ }^{1}$ Center for Research and Training in Skin Diseases and Leprosy, Tehran University of Medical Sciences, \\ 415 Taleghani Avenue, Tehran 14166 13675, Iran \\ ${ }^{2}$ Fasa University of Medical Sciences, Fasa, Iran
}

Correspondence should be addressed to Bardia Sadr, bsadr@farabi.tums.ac.ir

Received 26 October 2011; Accepted 17 November 2011

Academic Editors: S. Chimenti and H. Maibach

Copyright (C) 2012 Alireza Firooz et al. This is an open access article distributed under the Creative Commons Attribution License, which permits unrestricted use, distribution, and reproduction in any medium, provided the original work is properly cited.

\begin{abstract}
Background. Understanding the physiological, chemical, and biophysical characteristics of the skin helps us to arrange a proper approach to the management of skin diseases. Objective. The aim of this study was to measure 6 biophysical characteristics of normal skin (sebum content, hydration, transepidermal water loss (TEWL), erythema index, melanin index, and elasticity) in a normal population and assess the effect of sex, age, and body location on them. Methods. Fifty healthy volunteers in 5 age groups (5 males and females in each) were enrolled in this study. A multifunctional skin physiology monitor (Courage \& Khazaka electronic $\mathrm{GmbH}$, Germany) was used to measure skin sebum content, hydration, TEWL, erythema index, melanin index, and elasticity in 8 different locations of the body. Results. There were significant differences between the hydration, melanin index, and elasticity of different age groups. Regarding the locations, forehead had the highest melanin index, where as palm had the lowest value. The mean values of erythema index and melanin index and TEWL were significantly higher in males and anatomic location was a significant independent factor for all of 6 measured parameters. Conclusion. Several biophysical properties of the skin vary among different gender, age groups, and body locations.
\end{abstract}

\section{Introduction}

The skin is the largest multifunctional organ in the body. It functions as a protective physical barrier by absorbing UV radiation, preventing microorganism invasion and chemical penetration, and controlling the passage of water and electrolytes. The skin has a major role in thermoregulation of body, in addition to immunological, sensory, and autonomic functions [1]. Understanding the physiological, chemical, and biophysical characteristics of the skin helps us to arrange a proper approach to the management of skin diseases. However, it is critical to consider the influence of genetic and environmental factors on most of the skin characteristics.

Man et al. assessed the differences in the skin surface $\mathrm{pH}$, sebum content, and stratum corneum (SC) hydration at various ages and in both genders in a large Chinese population without skin diseases and concluded that these parameters vary with age, gender, and body site [2]. Marrakchi and Maibach established a preliminary map of the human face for 6 biophysical parameters in 9 locations and compared these various characteristics in different age groups [3].

The aim of this study is to assess the biophysical characteristics of normal skin with standardized experimental conditions in an Iranian population in order to compare with other studies.

\section{Materials and Methods}

2.1. Volunteers. Fifty healthy volunteers in 5 age groups were examined: $10-20,20-30,30-40,40-50$, and $50-60$ years old. There were 10 subjects in each group ( 5 females and 5 males). This study was approved by the ethics committee of 
Center for Research \& Training in Skin Diseases \& Leprosy and was performed according to the Declaration of Helsinki principles. All of the participants were instructed about the study and an informed consent was obtained from each one.

2.2. Methods. Eight body regions (forehead, cheek, nasolabial fold, neck, forearm, dorsal side of the hand, palm, and leg) were studied on their right sides. No skin care products were applied to the measured sites for at least 2 hours prior to the measurements. A small area of each location was wiped with ethanol 1 hour before the parameters were measured in a room at a temperature of $20-25^{\circ} \mathrm{C}$ and relative humidity of 30-40\%.

Skin sebum content, hydration, TEWL, erythema index, melanin index, and elasticity were measured with respective probes Sebumeter, Corneometer, TEWAmeter, Mexameter, and Cutometer (Courage \& Khazaka electronic $\mathrm{GmbH}$, Cologne, Germany). Sebumeter SM 815 uses the difference of light intensity through a plastic strip to indicate the amount of absorbed sebum. The sebum level is expressed in $\mu \mathrm{g} / \mathrm{cm}^{2}$ [4]. Corneometer CM 825 uses the high dielectric constant of water for analyzing the water-related changes in the electrical capacitance of the skin. It displays hydration measurements in system-specific arbitrary units [5]. A melanin index is calculated by Mexameter MX 18 from the strength of the absorbed and the reflected light at, respectively, 660 and $880 \mathrm{~nm}$. An erythema index is processed similarly at, respectively, 568 and $660 \mathrm{~nm}$ [6]. The measurement of TEWL by TEWAmeter TM 300 is based on the diffusion in an open chamber and is measured as $\mathrm{g} / \mathrm{m}^{2} / \mathrm{h}$ [7]. Cutometer MPA 580 pulls the targeted skin into the probe with a controlled vacuum pressure. Then the vertical deformation of the skin is measured and analyzed by computer softwares and is expressed arbitrarily [8].

2.3. Statistical Analysis. The data were analyzed with SPSS16 software (SPSS Inc. Chicago Ill). A mixed model ANOVA was used for comparison of data between study groups. In this analysis age (in five levels) and sex were defined as the fix effect factors. A variable which contained subject codes was defined as random effect factor. Also locations of the measurement (8 locations) were defined as repeated factors. To specify the relationship between the levels of random effects (8 locations), an unstructured covariance matrix was chosen. $P$ values $<0.05$ were considered significant.

\section{Results}

The mean and standard deviation of skin hydration, TEWL, melanin index, erythema index, sebum, and elasticity in both genders are shown in Table 1. Sex had an independent effect on TEWL, skin melanin index, and erythema index, but not on skin hydration, elasticity, or sebum.

The mean and standard deviation of these biophysical parameters in different age groups and body locations are shown in Tables 2 and 3, respectively. Age had a significant influence on skin hydration and melanin index $(P<0.05)$ and a marginally significant effect on elasticity $(P=0.05)$.
TABLE 1: The mean and standard deviation of skin hydration, TEWL, melanin index, erythema index, elasticity, and sebum according to gender.

\begin{tabular}{lcc}
\hline Variable & Male & Female \\
\hline Hydration & $48.42 \pm 22.12$ & $49.06 \pm 16.09$ \\
TEWL & $15.49 \pm 11.47$ & $9.52 \pm 7.36$ \\
Erythema index & $378.14 \pm 124.50$ & $303.63 \pm 100.73$ \\
Melanin index & $214.82 \pm 77.66$ & $176.82 \pm 58.42$ \\
Elasticity & $0.270 \pm 0.142$ & $0.273 \pm 0.121$ \\
Sebum & $60.39 \pm 74.52$ & $42.19 \pm 54.10$ \\
\hline
\end{tabular}

Anatomic location was a significant independent factor for all of 6 measured parameters.

\section{Discussion}

4.1. Hydration. Stratum corneum hydration has an important role in skin functions such as regulating epidermal proliferation, differentiation, and inflammation [2]. In this study skin hydration was higher in female subjects, but the difference was not statistically significant (Table 1). Ehlers et al. [9] reported that the skin of females and males was hydrated equally. No correlation was found between skin hydration and sex in another study [10].

As reported by Man et al. [2], we detected a significant relationship between skin hydration and age (Table 2). Marrakchi and Maibach [3] reported that the oldest individuals had the least hydrated skin. One of the factors causing reduced stratum corneum hydration in the older group is a decrease in natural moisturizers [2]. In a study about the effects of menopause on physiological characteristics of the skin, late menopausal women had higher skin hydration than peri/premenopausal women [11]. However, some other investigations found no relation between skin hydration and age $[10,12,13]$.

In concordance with our study, Shriner and Maibach [14] and also Marrakchi and Maibach [3] found out that neck had the most hydrated skin compared to the other parts of the face. This was due to high frequency conductance values of the neck [15]. Regarding ethnicity, it was reported that hydration of the skin and also the effect of age on hydration were influenced by ethnicity $[16,17]$. However, in other studies skin hydration showed no significant difference among ethnicities [18, 19]. Some of the dissimilarities between this study and others can be explained due to ethnical and environmental variations.

4.2. TEWL. Transepidermal water loss is used to assess skin water barrier function. We found out that TEWL was higher in males than that in females (Table 1). Males usually have more outdoor activities and their skins are more damaged. This is in contrast to the studies done by Ehlers et al. [9] who reported equal TEWL in both sexes. However, another research found no relation between TEWL and sex [10].

We found that TEWL was lower in the youngest and in the oldest subjects, but age did not show a significant effect 
TABLE 2: The mean and standard deviation of skin hydration, TEWL, melanin index, erythema index, sebum, and elasticity in 5 age groups.

\begin{tabular}{lccccc}
\hline & $10-20$ & $20-30$ & $30-40$ & $40-50$ & $50-60$ \\
\hline Hydration & $49.74 \pm 19.25$ & $47.08 \pm 16.61$ & $50.53 \pm 17.69$ & $53.34 \pm 20.78$ & $43.04 \pm 20.58$ \\
TEWL & $9.18 \pm 6.46$ & $14.90 \pm 12.59$ & $13.67 \pm 8.99$ & $14.64 \pm 11.08$ & $9.87 \pm 8.50$ \\
Melanin & $174.25 \pm 58.55$ & $235.95 \pm 82.15$ & $210.14 \pm 76.04$ & $181.10 \pm 57.90$ & $179.51 \pm 63.68$ \\
Erythema & $323.25 \pm 125.42$ & $370.36 \pm 113.74$ & $336.22 \pm 122.64$ & $337.62 \pm 113.42$ & $328.32 \pm 117.91$ \\
Sebum & $53.75 \pm 77.94$ & $50.10 \pm 51.81$ & $42.06 \pm 60.42$ & $66.71 \pm 73.42$ & $41.77 \pm 57.72$ \\
Elasticity & $.2561 \pm .1118$ & $.3025 \pm .1566$ & $.2887 \pm .1228$ & $.2803 \pm .1211$ & $.2345 \pm .1348$ \\
\hline
\end{tabular}

on TEWL. A negative correlation between age and TEWL has been reported in several studies [10, 20-22]. However, Marrakchi and Maibach found no correlation between these two parameters [3]. Also, Shriner and Maibach found no relation between TEWL and perceived age [14].

In this study, the palm and the leg had the highest and the lowest TEWL, respectively (Table 3). Palm is believed to be an exception. Despite the great thickness of the stratum corneum of the palm, it is the low amount of stratum corneum barrier lipids which causes the high level of TEWL on palm [15]. Marrakchi and Maibach [3] reported that TEWL was significantly higher in the nasolabial fold than the forehead. Tagami [15] showed that TEWL of forehead and the nasolabial fold were significantly higher than the cheek. On the other hand, Lopez et al. [22] and also Le Fur et al. [23] reported that TEWL of the cheek was significantly higher than that of the forehead. Variations in TEWL levels are due to different factors such as skin blood flow, skin temperature, the stratum corneum lipid contents, and the degree of corneocyte formation [3]. Moreover, our sample size, ethnicity, and methodological differences may have affected the results. Wesley and Maibach reported that TEWL was greater in black skin compared with white skin, but it was inconclusive in Asians [19]. Another study showed no difference in TEWL between Black, African, or Carribean Mixed-race and Caucaisan women [18].

4.3. Sebum. In this study, sex did not have a significant effect on sebum, although skin sebum content was higher in males (Table 1). It is known that sebum production correlates positively with testosterone levels in both sexes, through dehydroepiandrosterone in males and etiocholanolone in females [2]. Other studies also have shown that sebum levels were the same in both sexes $[9,10]$.

We did not find significant difference in skin sebum content among age groups (Table 2). Additionally, another study found no relation between skin sebum and age [10]. In a report from Switzerland [12], skin sebum level decreased with age. Furthermore, Ohta et al. [11] reported that skin sebum content is reduced after menopause in women. The differences may be due to sample size and ethnicity.

We found out that sebum secretion was the highest on the nasolabial fold and the lowest on the leg (Table 3). Also Lopez et al. [22] and Tagami [15] reported that skin sebum level was significantly higher in the forehead than that in the cheek. Another study found out that sebum level was the highest in the central areas of the face such as the nasolabial fold in young individuals. Some factors such as hormones, age, sex, and ethnicity could affect the sebum secretion; therefore, standardized experimental methods and conditions are required [3]. Castelo-Branco et al. Maibach reported that lipid contents were different in regarding ethnicity, but they were inconclusive [24]. In another study, the effects of ethnicity on skin lipid content were assessed but no significance was reached [19].

4.4. Skin Pigmentation. Melanin is one of the pigments which determine the skin color [25]. In our study, skin melanin index was significantly higher in males (Table 1 ). We also found out that subjects aged 20-30 years and 10-20 years had the highest and the lowest skin melanin index, respectively (Table 2). However, in a study which was done in China [13], no correlation was found between skin pigmentation and age. In our study, forehead was the most pigmented area, whereas the palm had the lowest skin melanin index (Table 3). This can be explained by the degree of sun exposure. A study which was conducted in Japan reported that individuals who lived in sun-exposed areas had higher skin melanin index compared to people who lived in less sun-exposed areas [26]. Hermanns et al. found out that the pattern of melanin index variation in different body parts was irrespective of the skin phototype and the dorsal forearm always had the highest melanin index [27].

4.5. Erythema Index. Quantification of erythema and melanin is useful for analysis of skin tests and management of skin diseases [6]. Personal factors (age, sex, race, anatomical site, skin surface properties), environmental factors (light conditions, temperature), and different procedures influence skin colour [28]. We found out that skin erythema index was higher in males than females (Table 1) but was not significantly different among age groups (Table 2). Regarding body location, the nasolabial fold had the highest erythema index. On the other hand, leg had the lowest skin erythema index (Table 3). In a study done in Belgium, 4 parts of the body were investigated in 137 normal individuals. They found out that the forehead had the maximum erythema index. Also they concluded that regional variability in erythema index was unpredictable [27]. Clarys et al. compared three skin color measurement instruments (Chromameter, Dermaspectrephotometer, and Mexameter) by evaluating several parameters such as erythema index in normal individuals. They found out that Chromameter was capable of measuring all colors, while the reflectance meters 
TABLE 3: The mean and standard deviation of skin hydration, TEWL, melanin index, erythema index, sebum, and elasticity according to body location.

\begin{tabular}{lcccccccc}
\hline & Forehead & Cheek & Nasolabial fold & Neck & Forearm & Dorsal & Palm & Leg \\
\hline Hydration & $53.54 \pm 16.49$ & $62.12 \pm 15.63$ & $38.19 \pm 18.02$ & $62.88 \pm 15.28$ & $51.00 \pm 15.92$ & $44.14 \pm 17.43$ & $40.47 \pm 18.47$ & $37.22 \pm 17.50$ \\
TEWL & $12.27 \pm 10.05$ & $9.57 \pm 7.22$ & $14.05 \pm 8.25$ & $10.47 \pm 9.23$ & $10.12 \pm 9.54$ & $9.86 \pm 8.84$ & $23.47 \pm 9.67$ & $9.68 \pm 9.52$ \\
Melanin & $228.16 \pm 66.48$ & $203.35 \pm 50.53$ & $202.29 \pm 54.01$ & $225.96 \pm 66.41$ & $193.31 \pm 70.34$ & $225.94 \pm 67.25$ & $98.98 \pm 41.75$ & $189.71 \pm 62.31$ \\
Erythema & $420.49 \pm 90.31$ & $399.80 \pm 91.12$ & $480.42 \pm 88.93$ & $373.27 \pm 97.31$ & $257.88 \pm 69.87$ & $331.94 \pm 62.23$ & $248 \pm 60.82$ & $205.00 \pm 63.52$ \\
Sebum & $95.65 \pm 51.38$ & $73.39 \pm 64.05$ & $136.98 \pm 72.33$ & $64.41 \pm 64.51$ & $18.45 \pm 37.88$ & $8.84 \pm 8.45$ & $9.82 \pm 10.11$ & $2.88 \pm 6.42$ \\
Elasticity & $.2901 \pm .1072$ & $.3040 \pm .0820$ & $.3057 \pm .1051$ & $.4528 \pm .1102$ & $.2783 \pm .0775$ & $.2280 \pm .0851 .1819 \pm .1339$ & $.1373 \pm .0685$ \\
\hline
\end{tabular}

(Mexameter and DermaSpectrometer) were suitable for evaluating the intensity of erythema and melanin-induced pigmentation [25].

4.6. Elasticity. Skin elasticity was higher in female subjects than in males (Table 1); however, the difference was not statistically significant. Also Ishikawa et al. [29] reported that skin elastic properties were not correlated with sex. On the other hand, the oldest age group had the least skin elasticity (Table 2), which is in concordance with another study done by Wendling and Dell'Acqua [12] The highest skin elasticity content was observed in the age group of 20-30 years. It was reported that skin collagen content showed a peak between the ages of 20 and 40 years and decreased between the ages of 40 and 60 years [24]. Some studies [29-31] found a negative correlation between forearm skin elastic properties and age in women. Sumino et al. [31] reported that skin elasticity decreased after menopause $0.55 \%$ per year; however, it increased by $5.2 \%$ after 12 months of hormone replacement therapy. It is known that severe disorganization of the elastic fiber network and decrease in the collagen fiber bundles occur with age. We found out that the neck and the leg had the most and the least skin elasticities, respectively (Table 3 ). In another study in which 4 parts of the body were examined (finger, hand, forearm, and chest), it was reported that skin elastic property of the chest was the highest [29]. These differences are mainly due to alterations in the elastic fiber network.

\section{Conclusion}

In this study we showed variations in several biophysical properties of the skin among different gender, age groups, and skin locations. These differences may be involved in the individual susceptibility to skin diseases. On the other hand, they should be considered in the formulation of skin care products. Genetic and environmental factors, methodology, and sample size might be involved in the variations in biophysical properties of skin reported in various studies.

\section{Acknowledgment}

This study was supported by research Grant no. 423/370 from the Center for Research \& Training in Skin Diseases \& Leprosy, Tehran University of Medical Sciences.

\section{References}

[1] C. B. Archer, "Functions of the skin," in Rook's Textbook of Dermatology, T. Burns, S. Breathnach, N. Cox, and C. Griffiths, Eds., vol. 1. 4.1., Blackwell, Oxford, UK, 2004.

[2] M. Q. Man, S. J. Xin, S. P. Song et al., "Variation of skin surface $\mathrm{pH}$, sebum content and stratum corneum hydration with age and gender in a large chinese population," Skin Pharmacology and Physiology, vol. 22, no. 4, pp. 190-199, 2009.

[3] S. Marrakchi and H. I. Maibach, "Biophysical parameters of skin: map of human face, regional, and age-related differences," Contact Dermatitis, vol. 57, no. 1, pp. 28-34, 2007.

[4] S. Y. Pande and R. Misri, "Sebumeter," Indian Journal of Dermatology, Venereology and Leprology, vol. 71, no. 6, pp. 444-446, 2005.

[5] L. C. Gerhardt, V. Strässle, A. Lenz, N. D. Spencer, and S. Derler, "Influence of epidermal hydration on the friction of human skin against textiles," Journal of the Royal Society Interface, vol. 5, no. 28, pp. 1317-1328, 2008.

[6] T. Yamamoto, H. Takiwaki, S. Arase, and H. Ohshima, "Derivation and clinical application of special imaging by means of digital cameras and Image $J$ freeware for quantification of erythema and pigmentation," Skin Research and Technology, vol. 14, no. 1, pp. 26-34, 2008.

[7] J. H. Shah, H. Zhai, and H. I. Maibach, "Comparative evaporimetry in man," Skin Research and Technology, vol. 11, no. 3, pp. 205-208, 2005.

[8] H. S. Ryu, Y. H. Joo, S. O. Kim, K. C. Park, and S. W. Youn, "Influence of age and regional differences on skin elasticity as measured by the Cutometer ${ }^{\circledR}$," Skin Research and Technology, vol. 14, no. 3, pp. 354-358, 2008.

[9] C. Ehlers, U. I. Ivens, M. L. Møller, T. Senderovitz, and J. Serup, "Females have lower skin surface $\mathrm{pH}$ than men: a study on the influence of gender, forearm site variation, right/left difference and time of the day on the skin surface $\mathrm{pH}$," Skin Research and Technology, vol. 7, no. 2, pp. 90-94, 2001.

[10] K. P. Wilhelm, A. B. Cua, and H. I. Maibach, "Skin aging: effect on transepidermal water loss, stratum corneum hydration, skin surface $\mathrm{pH}$, and casual sebum content," Archives of Dermatology, vol. 127, no. 12, pp. 1806-1809, 1991.

[11] H. Ohta, K. Makita, T. Kawashima, S. Kinoshita, M. Takenouchi, and S. Nozawa, "Relationship between dermatophysiological changes and hormonal status in pre-, peri-, and postmenopausal women," Maturitas, vol. 30, no. 1, pp. 55-62, 1998.

[12] P. A. Wendling and G. Dell'Acqua, "Skin biophysical properties of a population living in Valais, Switzerland," Skin Research and Technology, vol. 9, no. 4, pp. 331-338, 2003.

[13] A. E. Mayes, P. G. Murray, D. A. Gunn et al., "Ageing appearance in China: biophysical profile of facial skin and 
its relationship to perceived age," Journal of the European Academy of Dermatology and Venereology, vol. 24, no. 3, pp. 341-348, 2010.

[14] D. L. Shriner and H. I. Maibach, "Regional variation of nonimmunologic contact urticaria functional map of the human face," Skin Pharmacology, vol. 9, no. 5, pp. 312-321, 1996.

[15] H. Tagami, "Location-related differences in structure and function of the stratum corneum with special emphasis on those of the facial skin," International Journal of Cosmetic Science, vol. 30, no. 6, pp. 413-434, 2008.

[16] J. de Rigal, I. Des Mazis, S. Diridollou et al., "The effect of age on skin color and color heterogeneity in four ethnic groups," Skin Research and Technology, vol. 16, no. 2, pp. 168-178, 2010.

[17] S. Diridollou, J. De Rigal, B. Querleux, F. Leroy, and V. Holloway Barbosa, "Comparative study of the hydration of the stratum corneum between four ethnic groups: influence of age," International Journal of Dermatology, vol. 46, no. 1, pp. 11-14, 2007.

[18] C. Fotoh, A. Elkhyat, S. Mac, J. M. Sainthillier, and P. Humbert, "Cutaneous differences between Black, African or Caribbean Mixed-race and Caucasian women: biometrological approach of the hydrolipidic film," Skin Research and Technology, vol. 14, no. 3, pp. 327-335, 2008.

[19] N. O. Wesley and H. I. Maibach, "Racial (ethnic) differences in skin properties: the objective data," American Journal of Clinical Dermatology, vol. 4, no. 12, pp. 843-860, 2003.

[20] H. Kobayashi and H. Tagami, "Distinct locational differences observable in biophysical functions of the facial skin: with special emphasis on the poor functional properties of the stratum corneum of the perioral region," International Journal of Cosmetic Science, vol. 26, no. 2, pp. 91-101, 2004.

[21] H. Kobayashi and H. Tagami, "Functional properties of the surface of the vermilion border of the lips are distinct from those of the facial skin," British Journal of Dermatology, vol. 150, no. 3, pp. 563-567, 2004.

[22] S. Lopez, I. Le Fur, F. Morizot, G. Heuvin, C. Guinot, and E. Tschachler, "Transepidermal water loss, temperature and sebum levels on women's facial skin follow characteristic patterns," Skin Research and Technology, vol. 6, no. 1, pp. 3136, 2000.

[23] I. Le Fur, S. Lopez, F. Morizot, C. Guinot, and E. Tschachler, "Comparison of cheek and forehead regions by bioengineering methods in women with different self-reported 'cosmetic skin types,' Skin Research and Technology, vol. 5, no. 3, pp. 182-188, 1999.

[24] C. Castelo-Branco, F. Pons, E. Gratacós, A. Fortuny, J. A. Vanrell, and J. González-Merlo, "Relationship between skin collagen and bone changes during aging," Maturitas, vol. 18, no. 3, pp. 199-206, 1994.

[25] P. Clarys, K. Alewaeters, R. Lambrecht, and A. O. Barel, "Skin color measurements: comparison between three instruments: the Chromameter ${ }^{\circledR}$, the DermaSpectrometer ${ }^{\circledR}$ and the Mexameter ${ }^{\circledR}, "$ Skin Research and Technology, vol. 6, no. 4, pp. 230-238, 2000.

[26] G. G. Hillebrand, K. Miyamoto, B. Schnell, M. Ichihashi, R. Shinkura, and S. Akiba, "Quantitative evaluation of skin condition in an epidemiological survey of females living in northern versus southern Japan," Journal of Dermatological Science, vol. 27, no. 1, pp. S42-S52, 2001.

[27] J. F. Hermanns, L. Petit, T. Hermanns-Lê, and G. E. Piérard, "Analytic quantification of phototype-related regional skin complexion," Skin Research and Technology, vol. 7, no. 3, pp. 168-171, 2001.
[28] A. Fullerton, T. Fischer, A. Lahti, K.-P. Wilhelm, H. Takiwaki, and J. Serup, "Guidelines for measurement of skin colour and erythema. A report from the Standardization Group of the European Society of Contact Dermatitis," Contact Dermatitis, vol. 35, no. 1, pp. 1-10, 1996.

[29] T. Ishikawa, O. Ishikawa, and Y. Miyachi, "Measurement of skin elastic properties with a new suction device (I): relationship to age, sex and the degree of obesity in normal individuals," Journal of Dermatology, vol. 22, no. 10, pp. 713717, 1995.

[30] A. B. Cua, K. P. Wilhelm, and H. I. Maibach, "Elastic properties of human skin: relation to age, sex, and anatomical region," Archives of Dermatological Research, vol. 282, no. 5, pp. 283-288, 1990.

[31] H. Sumino, S. Ichikawa, M. Abe, Y. Endo, O. Ishikawa, and M. Kurabayashi, "Effects of aging, menopause, and hormone replacement therapy on forearm skin elasticity in women," Journal of the American Geriatrics Society, vol. 52, no. 6, pp. 945-949, 2004. 


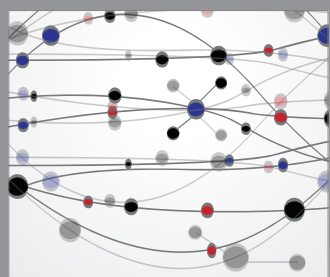

The Scientific World Journal
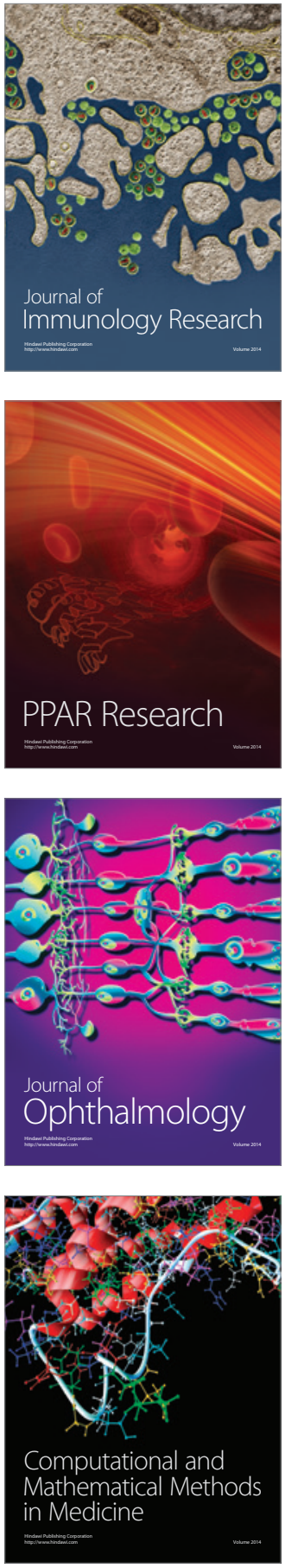

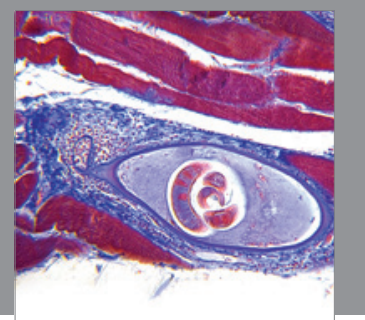

Gastroenterology

Research and Practice
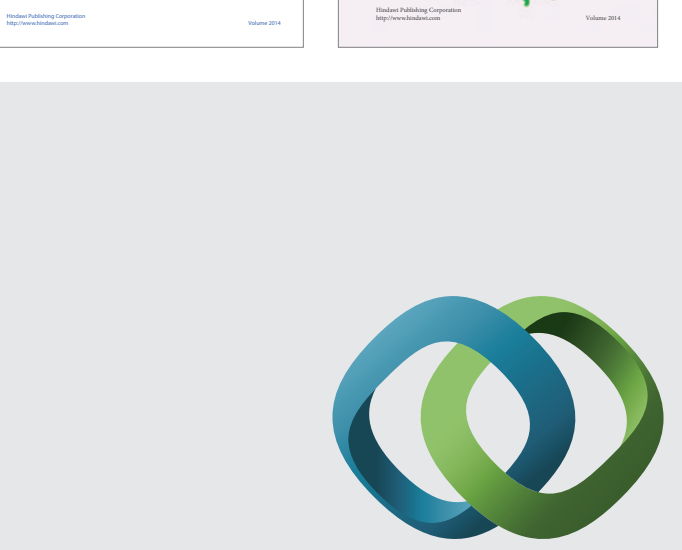

\section{Hindawi}

Submit your manuscripts at

http://www.hindawi.com
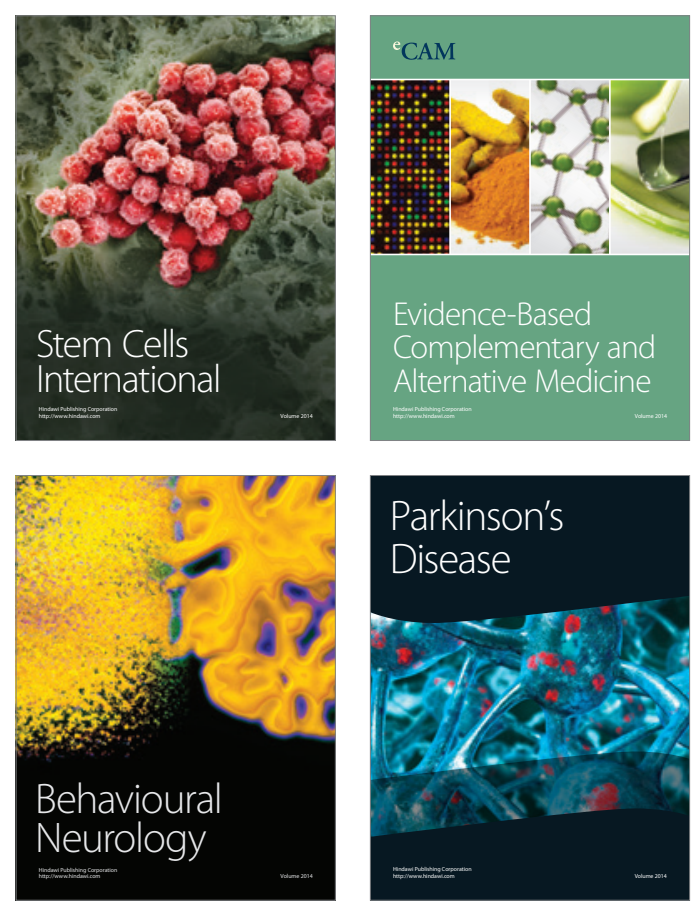

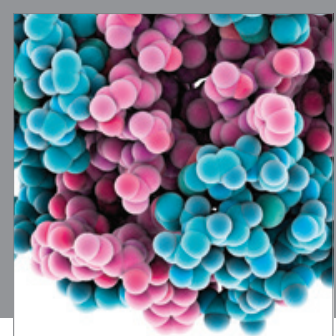

Journal of
Diabetes Research

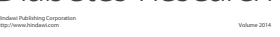

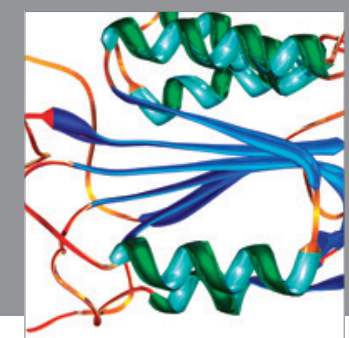

Disease Markers
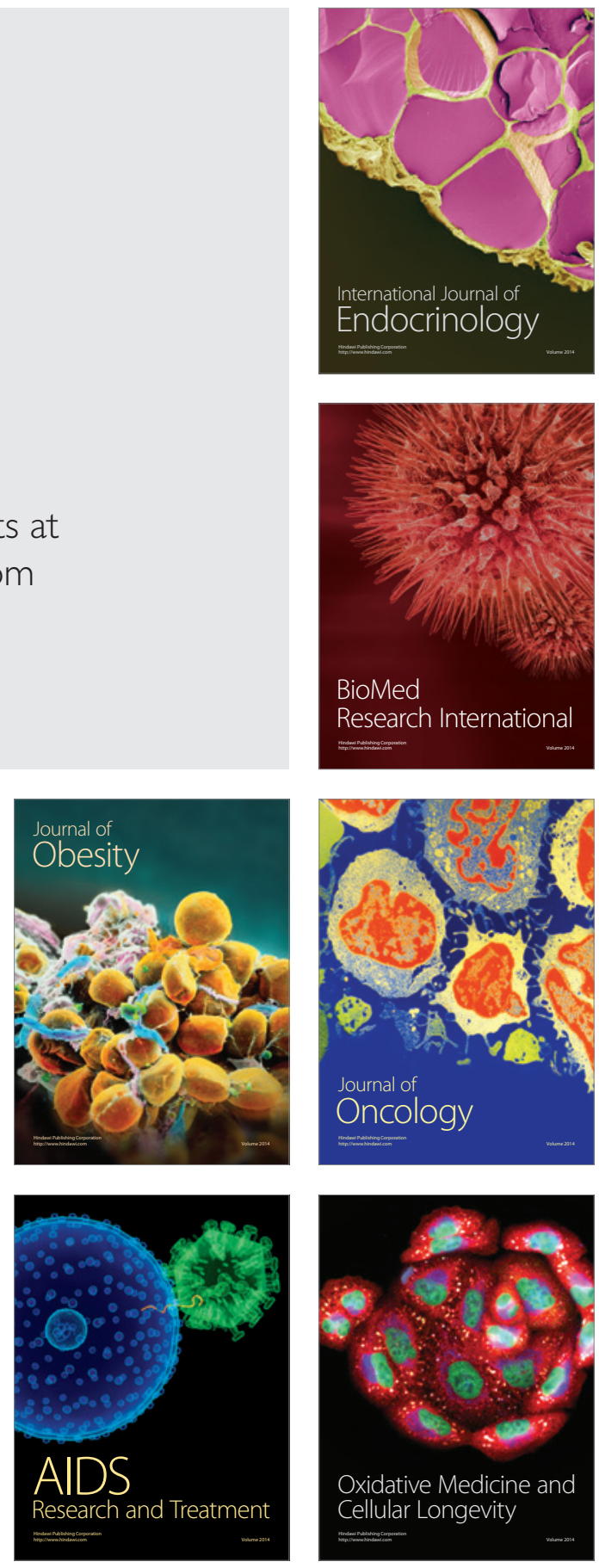